\title{
La alimentación en el adolescente
}

\section{Food consumption patterns among adolescents}

\author{
S.M. Palenzuela Paniagua ${ }^{1}$, A. Pérez Milena ${ }^{2}$, L.A. Pérula de Torres ${ }^{3}$, \\ J.A. Fernández García ${ }^{4}$, J. Maldonado Alconada ${ }^{5}$
}

\section{RESUMEN}

Fundamento. La adolescencia es un momento clave para la instauración de hábitos alimentarios saludables. El objetivo del trabajo fue conocer los hábitos alimentarios de los adolescentes y su relación con factores socio-familiares.

Material y métodos. Estudio observacional descriptivo, multicéntrico, mediante cuestionario anónimo sobre consumo de alimentos en la última semana, recogiendo edad/sexo del alumnado, estudios/ocupación laboral de padres/madres y tipo/ localización del colegio. La muestra comprendió 1.095 adolescentes de sexto de Educación Primaria de una provincia andaluza, elegidos mediante muestreo aleatorio polietápico diferenciando centros públicos/privados y de la provincia/capital.

Resultados. Fueron recogidas 1.005 encuestas válidas (99,25\% del total); media de edad 11,45 años $( \pm 0,59)$ y $53 \%$ varones. Consumo deficitario de lácteos (sólo dos terceras partes toman diariamente), pasta, frutas, verduras y hortalizas (consumo diario en un $30 \%$ ). Las legumbres se consumen semanalmente en el $64,5 \%$. El consumo de pescado se iguala al de carne, siendo más preferida la de ave; más de la mitad consumen embutidos diariamente. El aceite de oliva sigue siendo el más consumido. Hay un significativo consumo de alimentos con "calorías vacías" (comida rápida, dulces, refrescos). Mediante análisis multivariante (clúster) se comprueba la existencia de agrupaciones de alimentos saludables y no saludables, relacionado con el nivel social de los progenitores y el tipo de colegio.

Conclusiones. La dieta basada en la pirámide nutricional no es el patrón de alimentación en los adolescentes encuestados, con escaso consumo de lácteos, legumbres y frutas/verduras. Los alimentos se agrupan en patrones saludables o no saludables relacionados con la clase social familiar. Son necesarias estrategias sanitarias para modificar este consumo inadecuado.

Palabras clave. Adolescente. Alimentación. Encuesta nutricional. Clase social. Requerimientos nutricionales.

\begin{abstract}
Background. Adolescence is a critical time for the establishment of healthy eating habits. The objective was to analyze food consumption patterns among adolescents and their relationship with family and social factors.
\end{abstract}

Methods. Multicentre observational cross-sectional descriptive study using a food frequency questionnaire for the last week. It was answered anonymously. The adolescent's age/ gender, parents' studies/occupation and school's location/ type were included. The population sample was composed of 1,095 adolescents in sixth grade at primary schools from an Andalusian region. They were chosen by polietapic random sampling that distinguished between public/private and capital/provincial schools.

Results. 1,005 surveys were analyzed. The mean age is 11.45 (SD: 0.59). Fifty-three percent were male. The intake of dairy products (only two-thirds taken daily), pasta, fruit and vegetables (daily consumption of $30 \%$ ) is deficient. Sixty-four point five percent consume legumes weekly. Fish consumption is equal to meat, with a preference for poultry. More than half consume red meat daily. Olive oil is preferred. The intake of "empty calories" (fast food, candies, soft drink) is high. Through multivariate analysis the existence of clusters of healthy and unhealthy foods, related to the social status of the parents and the type of school, is proved.

Conclusions. A healthy diet based on the nutritional pyramid is not the consumption pattern in the adolescents surveyed. There is a low consumption of diary products, legumes, fruits and vegetables. There is a relationship between the social class of the family and consumption patterns (healthy and unhealthy). Health strategies are needed to modify such inappropriate consumption.

Key words. Adolescent. Dietary habits. Nutrition survey. Social class. Nutritional requirements.

\section{An. Sist. Sanit. Navar. 2014; 37 (1): 47-58}

1. Centro de Salud de Otero. Instituto Nacional de Gestión Sanitaria (Ceuta). España. Unidad Docente de MFyC de Ceuta.

2. Centro de Salud el Valle. Unidad Docente de MFyC de Jaén.

3. Unidad Docente de Medicina Familiar y Comunitaria de Córdoba. Instituto Maimónides de Investigación Biomédica de Córdoba (IMIBIC)/Hospital Universitario Reina Sofía/ Universidad de Córdoba.

4. Centro de Salud de Villarrubia (Córdoba). España. Instituto Maimónides de Investigación Biomédica de Córdoba (IMIBIC)/Hospital Universitario Reina Sofía/Universidad de Córdoba.

5. Centro de Salud de Tarajal (Ceuta). España. Unidad Docente de MFyC de Ceuta.

\section{Correspondencia:}

Sara Palenzuela Paniagua.

E-mail: sarapalen@hotmail.com

Recepción: 30 de julio de 2013

Aceptación provisional: 16 de octubre de 2013

Aceptación definitiva: 20 de noviembre de 2013 


\section{INTRODUCCIÓN}

Más de 300 millones de personas en el mundo son obesos; en Europa afecta al 12\% de los hombres y al $18 \%$ de las mujeres ${ }^{1}$. Uno de cada 10 niños entre 2 y 17 años tiene obesidad y dos sobrepeso ${ }^{2}$. Las enfermedades que aparecen en la segunda mitad de la vida dependen en buena medida de la alimentación que se ha seguido durante las primeras etapas y se ha demostrado que existe una relación directa entre ésta y la morbimortali$\mathrm{dad}^{3}$. Según la OMS, los principales factores determinantes de la salud están ligados a la alimentación y a la práctica de actividad física. Así, llevar una alimentación equilibrada, realizar actividad física de manera habitual y mantener un peso adecuado a lo largo de la vida es el medio para protegerse de la mayoría de las enfermedades crónicas ${ }^{3}$. Sin embargo, los hábitos alimentarios del mundo occidental se caracterizan por un consumo excesivo, superior en términos generales a las ingestas recomendadas en cuanto a energía y nutrientes para el conjunto de la población y, cualitativamente, por un tipo de dieta rica en proteínas y grasas de origen animal ${ }^{4}$.

La dieta española sigue, en general, el patrón antes indicado si bien tiene como ventaja la presencia de un mayor consumo de vegetales, legumbres, frutas y el uso de aceites de origen vegetal ${ }^{4-6}$. La dieta mediterránea, basada en un bajo porcentaje de grasas saturadas y proteínas de origen animal y alta en hidratos de carbono complejos, fibras y sustancias antioxidantes, garantiza un aporte calórico de nutrientes en cantidades suficientes y proporciones adecuadas, y contribuye a la prevención de enfermedades cardiovasculares $^{3,6}$. Pese a ello, España se presenta como uno de los países con mayor tasa de obesidad infantil ${ }^{1}$, llegando a cifras del $17 \%$ de los adolescentes ${ }^{7}$.

El objetivo del estudio fue recoger información sobre la alimentación de los escolares por medio de un cuestionario de frecuencia de consumo de alimentos y valorar si ésta se ajusta al patrón recomendado en la pirámide nutricional. Este tipo de encuesta constituye un instrumento viable con adecuado grado de fiabilidad para la aproximación al conocimiento de la ingesta real de los hábitos alimentarios ${ }^{8-10}$. La identificación de patrones de consumo y poblaciones de ries- go ayudará a elaborar mejores programas de promoción de la salud.

\section{MATERIAL Y MÉTODOS}

Se diseñó un estudio observacional descriptivo mediante encuesta. La población de estudio fue el total del alumnado escolarizado en sexto de Educación Primaria de la provincia de Córdoba durante el curso escolar 2005/2006, siendo en total 8.944 sujetos. Como criterios de exclusión se indican la negativa del escolar/progenitores a responder a la encuesta y/o padecer alguna limitación que impidiera al alumno comprender o rellenar el cuestionario. Los participantes fueron escogidos mediante muestreo aleatorio polietápico en dos fases, estratificado y por conglomerados, teniendo en cuenta las características de los centros escolares (público/privado/concertado) y su ubicación geográfica (capital/provincia). Se incluyeron a todos los alumnos de $6^{\circ}$ de Educación Primaria de los colegios elegidos. El tamaño muestral fue de 1.005 escolares (precisión $\pm 0,03$, nivel de confianza $95 \%$, proporción estimada del $50 \%$, tasa de no respuesta $6 \%$ y posible absentismo 10\%).

La recogida de datos se realizó mediante un cuestionario anónimo estructurado y precodificado, validado y basado en el utilizado para el "Estudio de los hábitos de los escolares en relación con la salud (HBSC)" ${ }^{11}$. Éste recoge variables socio-demográficas (edad/ sexo/clase social y localización/carácter del colegio). Las diferentes categorías de clase social se definen en la tabla 1. Ésta se obtuvo siguiendo la propuesta de la Sociedad Española de Epidemiologia que está basada en las ocupaciones laborales ${ }^{12}$. También se recoge la frecuencia de consumo semanal de diferentes tipos de alimentos mediante cinco opciones categorizadas (varias veces al día/una vez al día/alguna vez a la semana/rara vez/nunca). Los alimentos se agrupan en siete grupos según su composición de hidratos de carbono, proteínas y grasas, siguiendo las directrices del Ministerio de Sanidad (España) y la Sociedad Española de Nutrición Comunitaria ${ }^{13}$. La encuesta se pilotó en una muestra de 30 escolares de 11-14 años de un colegio, obteniendo una adecuada fiabilidad intraobservador ${ }^{14}$. Se rechazan los cuestionarios cumplimentados en menos de un $75 \%$ de total. 
Tabla 1. Estratificación de la clase social según la ocupación laboral (modificada de la Sociedad Española de Epidemiología).

\begin{tabular}{clcc}
\hline $\begin{array}{c}\text { Clase } \\
\text { social }\end{array}$ & \multicolumn{1}{c}{ Ocupación } & Padre & Madre \\
\hline I & $\begin{array}{l}\text { Directivos de la administración y empresas de 10 o más asalariados. Altos funcio- } \\
\text { narios. Profesiones asociadas a titulaciones de segundo y tercer ciclo universitario. }\end{array}$ & $6 \%$ & $2,4 \%$ \\
\hline II & $\begin{array}{l}\text { Directivos de empresas con menos de 10 asalariados. Profesiones asociadas a una } \\
\text { titulación de primer ciclo universitario. Otros técnicos. Artistas y deportistas. }\end{array}$ & $8,5 \%$ & $10 \%$ \\
\hline & Empleados de tipo administrativo y profesionales de apoyo a la gestión admi- & \\
nill & $\begin{array}{l}\text { nistrativa y financiera. Personal de los servicios de protección y seguridad. } \\
\text { Trabajadores por cuenta propia. Supervisores de trabajadores manuales. }\end{array}$ & $14,3 \%$ & $12,8 \%$ \\
\hline IV & $\begin{array}{l}\text { Trabajadores manuales cualificados de la industria, comercio y servicios. } \\
\text { Trabajadores manuales semicualificados de la industria, comercio y servicios. }\end{array}$ & $53,8 \%$ & $12,3 \%$ \\
\hline V & Trabajadores no cualificados. & $12 \%$ & $17,7 \%$ \\
\hline Vla & Miembros del clero y fuerzas armadas. & $5,2 \%$ & $0 \%$ \\
\hline VIb & Amas de casa. & $0 \%$ & $40,4 \%$ \\
\hline VIc & Mal especificado/no consta. & $0 \%$ & $4 \%$ \\
\hline
\end{tabular}

Los datos fueron analizados utilizando el paquete estadístico SPSS 13.0 (SPSS Inc., Chicago, IL). Tras comprobar la normalidad de los datos se realizó un estudio descriptivo calculando porcentajes y frecuencias absolutas, así como las medias con sus correspondientes desviaciones típicas. Se valoraron las diferencias entre variables mediante el test de la $\mathrm{Ji}^{2}$ con un nivel de significación de $\mathrm{p}<0,05$.

Se hizo un análisis multivariante tipo clúster encaminado a analizar la pertinencia a grupos de las variables que medían el grado de consumo de distintos alimentos, y así discriminar distintos perfiles taxonómicos de consumo. Con ello se pretende analizar si existen alimentos que se puedan encuadrar dentro de unos determinados perfiles de consumo. Mediante este tipo de análisis multivariante se pretende buscar unas clases, de tal modo que cada observación difiera, de acuerdo a un cierto criterio, lo menos posible de las demás observaciones que pertenecen a la misma clase, partiendo de observaciones no clasificadas previamente. Estas clases o grupos se definen mediante el cálculo de distancias o similitudes, a partir de las variables que se consideran adecuadas para ello. Para una mejor comprensión se representó un dendograma (en forma de árbol lógico), que indica la distancia a la que se han producido las uniones y las variables implicadas en cada paso, lo que permite evidenciar la existencia o no de dos o más grupos.

Los grupos de alimentos hallados se compararon con la distribución de los mismos en la pirámide nutricional para este grupo de edad ${ }^{15}$ siendo los más recomendables aquellos que son ricos en proteínas (carnes/pescados) y en hidratos de carbono, fibra y micronutrientes (pastas/ legumbres/pan integral/cereales/verduras/ frutas/zumos), los lácteos (leche/yogurt/ queso) y otros, como el aceite de oliva (grasas), y pan. Como alimentos menos recomendables quedan los alimentos grasos (embutidos/comida rápida como hamburguesas y perritos calientes/aceite de girasol/frutos secos), los alimentos ricos en hidratos de carbono de rápida absorción y grasas saturadas (chocolate/golosinas/ dulces/bolsas de fritos/helados/grasas industriales) y otros alimentos como los estimulantes (café/té).

El estudio fue aprobado por el Comité de Ética en Investigación Clínica del Hospital Reina Sofía (Córdoba), garantizando el anonimato de las respuestas y solicitando un consentimiento informado a progenitores/tutores y adolescentes para su participación. 


\section{RESULTADOS}

Se obtuvo como muestra un total de 1.005 encuestas de 27 colegios en 19 localidades distintas, tras perder a 83 alumnos que no acudieron a clase y descartar 7 encuestas mal cumplimentadas. Se encuestaron 531 hombres (52,9\%) y 474 mujeres $(47,2 \%)$ con una media de edad de 11,45 años \pm 0,591-DT- (límites 11-14 años). Los resultados de la clase social se muestran en la tabla 1, predominando los trabajados manuales cualificados entre los padres y las labores domésticas entre las madres. En la tabla 2 se muestran los principales resultados del consumo de los grupos de alimentos estudiados y en las figuras 1 y 2 la relación de la frecuencia de consumo de determinados alimentos con la clase social y el tipo de colegio.

Tabla 2. Distribución de frecuencias de consumo según las recomendaciones para la frecuencia de uso de cada grupo de alimentos.

\begin{tabular}{|c|c|c|c|c|c|}
\hline & & $>1 \mathrm{vez} /$ día & Diaria & Semanal & Rara vez / Nunca \\
\hline \multicolumn{6}{|c|}{ Grupos de alimentos de consumo diario } \\
\hline \multirow{4}{*}{$\begin{array}{l}\text { Cereales y } \\
\text { derivados }\end{array}$} & Cereales & $9,4 \%$ & $27,1 \%$ & $46,7 \%$ & $16,8 \%$ \\
\hline & $\begin{array}{l}\text { Pasta } \\
\end{array}$ & $4,9 \%$ & $11,5 \%$ & $68,9 \%$ & $14,6 \%$ \\
\hline & Pan blanco & $35,8 \%$ & $32,3 \%$ & $18,4 \%$ & $12,6 \%$ \\
\hline & Pan integral & $5,7 \%$ & $8,9 \%$ & $12,5 \%$ & $71,9 \%$ \\
\hline \multicolumn{2}{|c|}{ Verduras y hortalizas } & $12,1 \%$ & $26,7 \%$ & $43,0 \%$ & $18,0 \%$ \\
\hline \multicolumn{2}{|l|}{ Frutas } & $42,0 \%$ & $32,4 \%$ & $16,1 \%$ & $9,4 \%$ \\
\hline \multicolumn{2}{|c|}{ Legumbres y tubérculos } & $5,1 \%$ & $22,0 \%$ & $64,5 \%$ & $8,2 \%$ \\
\hline \multirow{3}{*}{ Leche y derivados } & Leche & $67,3 \%$ & $25,9 \%$ & $2,6 \%$ & $4,0 \%$ \\
\hline & Queso & $21,3 \%$ & $26,1 \%$ & $28,8 \%$ & $23,7 \%$ \\
\hline & Yogurt & $40,6 \%$ & $35,5 \%$ & $16,7 \%$ & $7,0 \%$ \\
\hline \multicolumn{2}{|l|}{ Aceite oliva } & $27,5 \%$ & $33,1 \%$ & $24,8 \%$ & $10,8 \%$ \\
\hline \multicolumn{6}{|c|}{ Grupos de alimentos de consumo semanal } \\
\hline \multicolumn{2}{|l|}{ Carnes ave } & $4,3 \%$ & $12,1 \%$ & $63,6 \%$ & $19,9 \%$ \\
\hline \multicolumn{2}{|l|}{ Pescado azul } & $5,0 \%$ & $15,5 \%$ & $62,8 \%$ & $16,5 \%$ \\
\hline \multicolumn{2}{|l|}{ Pescado blanco } & $3,7 \%$ & $9,7 \%$ & $62,0 \%$ & $25,9 \%$ \\
\hline \multicolumn{2}{|l|}{ Huevos } & $4,9 \%$ & $17,2 \%$ & $68,3 \%$ & $9,3 \%$ \\
\hline \multicolumn{2}{|l|}{ Legumbres } & $5,1 \%$ & $22,0 \%$ & $64,5 \%$ & $8,2 \%$ \\
\hline \multicolumn{6}{|c|}{ Grupos de alimentos de consumo ocasional } \\
\hline \multicolumn{2}{|c|}{ Embutidos y fiambres } & $14,7 \%$ & $37,4 \%$ & $36,1 \%$ & $11,6 \%$ \\
\hline \multicolumn{2}{|l|}{ Carnes rojas } & $4,2 \%$ & $14,0 \%$ & $64,4 \%$ & $17,4 \%$ \\
\hline \multicolumn{2}{|l|}{ Frutos secos } & $9,5 \%$ & $14,4 \%$ & $43,9 \%$ & $32,0 \%$ \\
\hline \multicolumn{2}{|l|}{ Aceite girasol } & $9,1 \%$ & $15,6 \%$ & $27,7 \%$ & $47,5 \%$ \\
\hline \multicolumn{2}{|l|}{ Comida rápida } & $3,7 \%$ & $6,4 \%$ & $41,3 \%$ & $48,6 \%$ \\
\hline \multicolumn{2}{|l|}{ Refrescos } & $27,7 \%$ & $23,4 \%$ & $31,5 \%$ & $17,5 \%$ \\
\hline \multicolumn{2}{|l|}{ Zumos } & $35,9 \%$ & $31,0 \%$ & $22,6 \%$ & $10,6 \%$ \\
\hline \multicolumn{2}{|l|}{ Café/té } & $2,0 \%$ & $5,1 \%$ & $10,4 \%$ & $81,8 \%$ \\
\hline \multicolumn{2}{|l|}{ Chocolate } & $8,6 \%$ & $16,1 \%$ & $45,2 \%$ & $29,9 \%$ \\
\hline \multicolumn{2}{|l|}{ Dulces } & $8,5 \%$ & $15,8 \%$ & $43,3 \%$ & $43,3 \%$ \\
\hline \multicolumn{2}{|l|}{ Golosinas } & $18,0 \%$ & $20,3 \%$ & $42,1 \%$ & $19,6 \%$ \\
\hline \multicolumn{2}{|l|}{ Bolsas fritos } & $14,4 \%$ & $19,4 \%$ & $45,5 \%$ & $20,6 \%$ \\
\hline \multicolumn{2}{|l|}{ Helados } & $14,3 \%$ & $18,9 \%$ & $42,1 \%$ & $24,0 \%$ \\
\hline
\end{tabular}




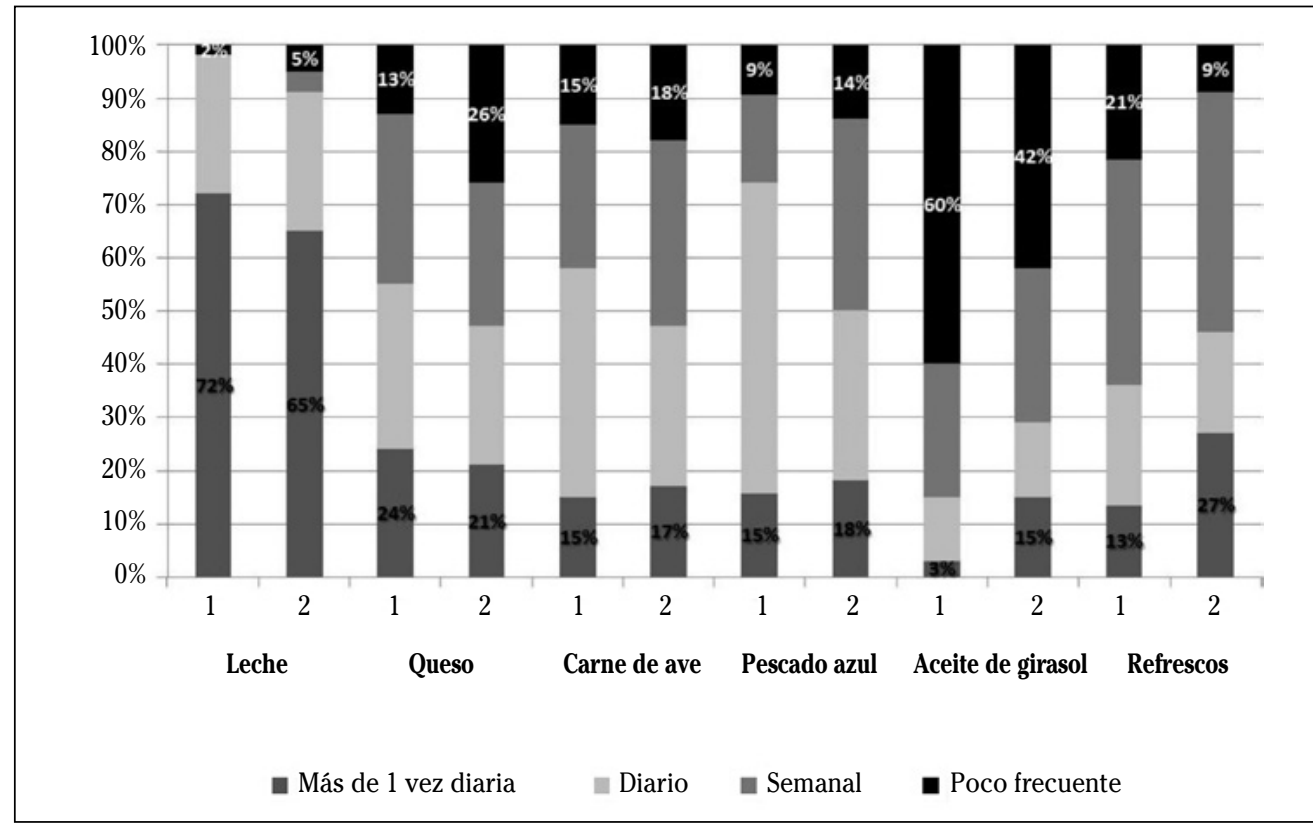

Figura 1. Relación entre el tipo y la distribución de frecuencias de alimentos consumidos con la clase social de la familia de origen del alumnado.

Clases sociales: $1=\mathrm{I}+\mathrm{II}+\mathrm{III}, 2=\mathrm{IV}+\mathrm{V}+\mathrm{VI}$. Diferencias significativas (clases sociales 1 y 2 ) en todos los alimentos con $\mathrm{p}<0,05$.

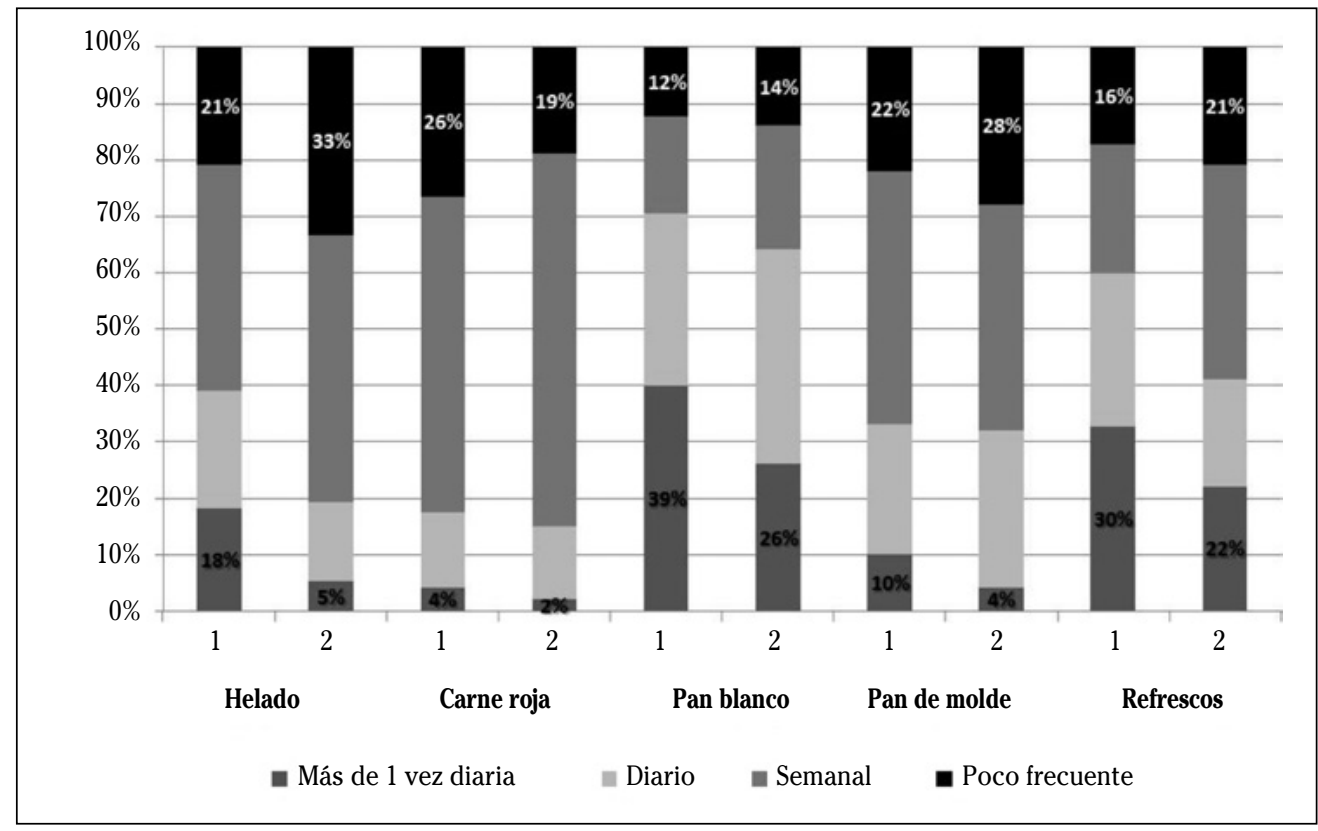

Figura 2. Relación entre el tipo y la distribución de frecuencias de alimentos consumidos con el tipo de colegio donde cursa estudios el alumnado.

Carácter del centro escolar: 1=Público, 2=Privado/concertado. Diferencias significativas en todos los alimentos con p<0,05. 
Dentro del grupo de lácteos, casi el 70\% de los alumnos toman leche varias veces al día, mientras que la cuarta parte casi nunca consumen queso. El consumo de lácteos es más frecuente cuando las madres pertenecen a las clases sociales I y II ( $\mathrm{p}=0,005$ $\left.\mathrm{Ji}^{2}: 25,081\right)$, mientras que el alumnado de clase social IV/V consumen más helados $\left(\mathrm{p}<0,001 \mathrm{Ji}^{2}: 47,686\right)$. Hay mayor toma de de yogurt y helados en los colegios públicos de la provincia $\left(\mathrm{p}<0,05 \mathrm{Ji}^{2}: 47,391\right)$.

En el grupo de carnes y pescados, se comprueba una mayor apetencia por los embutidos y carnes (el 14,7\% toman más de una vez al día embutidos) mientras que el pescado se consume de forma más ocasional (25\% lo toman rara vez o nunca). La carne roja y los embutidos son más frecuentemente consumidas por aquellos alumnos de colegios públicos $(\mathrm{p}=0,001$ $\left.\mathrm{Ji}^{2}: 13,187\right)$ mientras que la ingesta de pescado azul es más frecuente en las niñas $\left(\mathrm{p}=0,019 \mathrm{Ji}^{2}: 13,491\right)$ y en los mayores de 13 años $\left(\mathrm{p}=0,005 \mathrm{Ji}^{2}: 12,241\right)$. El consumo de carne y pescado azul es poco frecuente en las clases sociales IV/V ( $>20 \%$ en ambos casos, $\left.\mathrm{p}<0,05 \mathrm{Ji}^{2}: 35,139\right)$.

En el grupo de legumbres y frutos secos, el $64,5 \%$ consume legumbres de forma semanal y una quinta parte toma frutos secos a diario. La frecuencia de consumo de frutos secos es superior en niños $(\mathrm{p}=0,003$ $\left.\mathrm{Ji}^{2}: 14,282\right)$ y en asistentes a colegios públi$\cos \left(\mathrm{p}=0,032 \mathrm{Ji}^{2}: 12,250\right)$.

Del grupo de verduras, hortalizas y frutas destaca su consumo esporádico, mientras que un $43 \%$ realiza una ingesta semanal de verduras, casi la quinta parte lo hacen rara vez o nunca. Solo un $42,0 \%$ de los alumnos toman fruta de forma diaria, mientras que el $10 \%$ no toma nunca o casi nunca. El consumo diario de frutas es un $10 \%$ inferior en los niños de 13 años $\left(\mathrm{p}=0,001 \mathrm{Ji}^{2}: 23,056\right)$.

El alimento más utilizado dentro del grupo de cereales, pasta y pan es el pan blanco, consumido diariamente por el $68,1 \%$ de los adolescentes encuestados a diferencia del pan integral y los cereales (sobre el 70\% los consumen rara vez o nunca). La pasta se consume con más frecuencia con la edad $\left(\mathrm{p}=0,001 \mathrm{Ji}^{2}: 29,133\right)$ y más con madres de clase social IV/V/VI ( $\mathrm{p}=0,013$ $\left.\mathrm{Ji}^{2}: 29,646\right)$; junto con el pan blanco y el pan de molde, su uso es más frecuente en los colegios públicos $\left(\mathrm{p}<0,05 \mathrm{Ji}^{2}: 15,711\right)$. Por otro lado, el consumo del pan de molde es más frecuente en los alumnos de la capital $\left(\mathrm{p}<0,0001 \mathrm{Ji}^{2}: 17,776\right)$.

El aceite de oliva es el tipo de aceite más empleado en el grupo de las grasas (27,5\% lo consume más de una vez al día frente al 8,3\% del aceite de girasol), sobre todo entre los escolares de la provincia $\left(\mathrm{p}<0,0001 \mathrm{Ji}^{2}: 23,180\right)$. Un $33 \%$ lo usa de forma diaria, un $25 \%$ semanal y sólo un $11 \%$ lo usa esporádicamente. El aceite de girasol es más usado en las clases sociales IV/V/VI $\left(\mathrm{p}=0,013 \mathrm{Ji}^{2}: 29,646\right)$.

El último grupo está formado por bebidas, bolsas de fritos y golosinas. La mayor frecuencia de consumo de estos productos se centra en los varones $\left(\mathrm{p}=0,001 \mathrm{Ji}^{2}: 16,160\right)$, alumnos de mayor edad ( $\left.\mathrm{p}=0,013 \mathrm{Ji}^{2}: 16,113\right)$, centros públicos $\left(\mathrm{p}=0,007 \mathrm{Ji}^{2}: 12,231\right)$ y de clase social IV/V $\left(\mathrm{p}=0,023 \mathrm{Ji}^{2}: 27,761\right)$. El consumo de comida rápida, como hamburguesas o perritos calientes, ocurre de forma diaria en un $10 \%$ de los encuestados y de forma semanal en el $41 \%$ (Tabla 2). Hay diferencias en la frecuencia del consumo de este tipo de comida en función del sexo (más frecuente en hombres), la edad (superior cuanta mayor edad), el tipo de colegio (en públicos más que en concertados) y la clase social (mayor presencia en niveles más bajos) (Tabla 3). Más de la mitad de los adolescentes toman refrescos de forma diaria y la ingesta de golosinas se hace en más de una ocasión al día en el $18 \%$ de los adolescentes estudiados. Existe una relación entre el uso de comida rápida y la pertenencia a clases sociales englobadas en las categorías IV, V y VI (Tabla 3).

Mediante el análisis de clúster y su representación por medio del dendograma (Figura 3) se analiza la existencia de agrupaciones de alimentos que permiten comprobar la existencia de un patrón de consumo en función de la cercanía de los alimentos estudiados en la encuesta. El dendograma permite la formulación de los siguientes resultados: las carnes, los 
pescados, el pan blanco y el aceite de oliva se combinan en un clúster común con una distancia relativamente pequeña. Los lácteos, los zumos y las frutas por un lado; el pan de molde, el integral, los cereales y las pastas, por otro y, las verduras y legumbres en un tercero, se agrupan en tres clúster relativamente homogéneos. Todos ellos se combinan en un clúster común que coinciden en sus características de ser los alimentos considerados como "más saludables". Por el contrario, en el otro brazo del dendograma y más alejados, se encuentran los alimentos "menos saludables". Dentro de este grupo podemos hacer una subdivisión en 4 grupos según la distancia en la que se encuentran del resto, así: los frutos secos y helados conformarían un grupo; el aceite de girasol, los embutidos, los huevos y la comida rápida otro; los refrescos, el café y el té, un tercero; y finalmente, los dulces, chocolates, bolsas de fritos y golosinas un último subclúster. Por lo tanto, se constata una partición en dos clúster que agrupan, por una parte, los alimentos más saludables que se encuentran en la base de la pirámide alimentaria $\mathrm{y}$, por otro, los alimentos menos recomendables.

Tabla 3. Frecuencia del consumo de comida rápida según la edad y el sexo del menor, el carácter del centro escolar, y la clase social del padre y de la madre

\begin{tabular}{|c|c|c|c|c|c|}
\hline & & \multicolumn{3}{|c|}{ Frecuencia del consumo de comida rápida } & \multirow{2}{*}{ Estadístico } \\
\hline & & Diario & Semanal & Rara vez / Nunca & \\
\hline \multirow{2}{*}{ Sexo } & Hombre & 12 & 45 & 43 & \multirow{2}{*}{$\begin{array}{c}\mathrm{Ji}^{2}: 16,160 \\
\mathrm{p}=0,001\end{array}$} \\
\hline & Mujer & 8 & 37 & 55 & \\
\hline \multirow{3}{*}{ Edad } & 11 años & 7 & 42 & 50 & \multirow{3}{*}{$\begin{array}{c}\mathrm{Ji}^{2}: 16,113 \\
\mathrm{p}=0,013\end{array}$} \\
\hline & 12 años & 14 & 39 & 48 & \\
\hline & $\geq 13$ años & 19 & 47 & 35 & \\
\hline \multirow{2}{*}{$\begin{array}{c}\text { Carácter } \\
\text { del centro }\end{array}$} & Público & 11,6 & 42,8 & 45,5 & \multirow{2}{*}{$\begin{array}{c}\mathrm{Ji}^{2}: 12,231 \\
\mathrm{p}=0,007\end{array}$} \\
\hline & Concertado & 6,2 & 6,2 & 56,8 & \\
\hline \multirow{6}{*}{$\begin{array}{l}\text { Clase social } \\
\text { del padre }\end{array}$} & $\mathbf{I}$ & 1,7 & 32,2 & 66,1 & \multirow{6}{*}{$\begin{array}{c}\mathrm{Ji}^{2}: 27,761 \\
\mathrm{p}=0,023\end{array}$} \\
\hline & II & 3,5 & 36,5 & 60 & \\
\hline & III & 7,2 & 39,6 & 53,2 & \\
\hline & IV & 11 & 42,3 & 46,5 & \\
\hline & $\mathbf{V}$ & 15,4 & 46,2 & 38,5 & \\
\hline & VI & 0 & 62,5 & 37,5 & \\
\hline \multirow{6}{*}{$\begin{array}{l}\text { Clase social } \\
\text { de la madre }\end{array}$} & I & 0 & 29,2 & 70,8 & \multirow{6}{*}{$\begin{array}{c}\mathrm{Ji}^{2}: 30,765 \\
\mathrm{p}=0,009\end{array}$} \\
\hline & II & 2,0 & 36,6 & 61,4 & \\
\hline & III & 8,9 & 40,3 & 50 & \\
\hline & IV & 13,8 & 43,1 & 43,1 & \\
\hline & $\mathbf{V}$ & 12,1 & 47,4 & 40,5 & \\
\hline & VI & 10,9 & 40,4 & 48,6 & \\
\hline
\end{tabular}




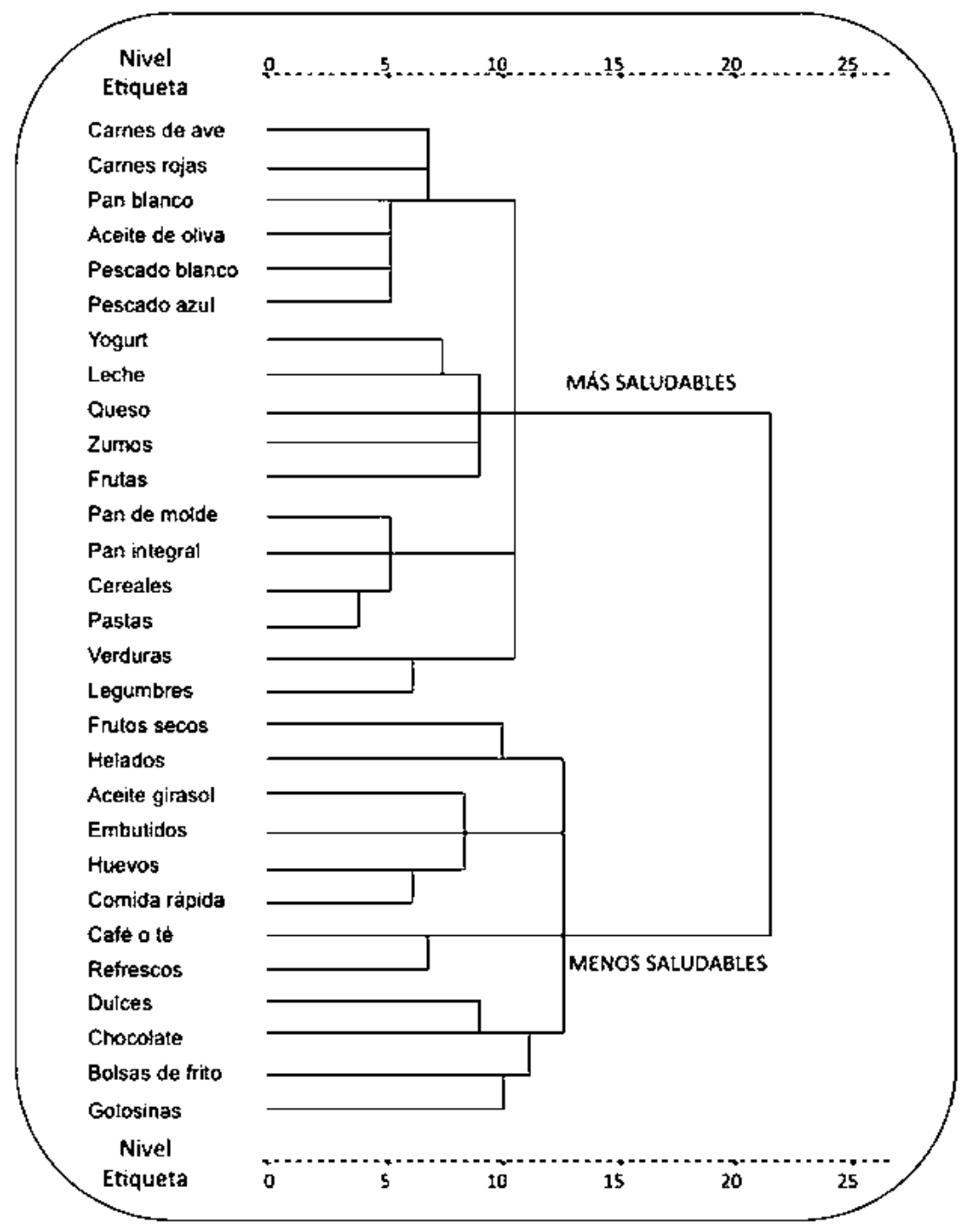

Figura 3. Dendograma del consumo de alimentos.

\section{DISCUSIÓN}

La alimentación durante la infancia y preadolescencia tiene una gran trascendencia en la proyección de la calidad de vida del adulto. En los resultados obtenidos en este trabajo, la dieta seguida por los escolares encuestados presenta ciertas desviaciones respecto a las recomendaciones dietéticas para los adolescentes, muy en consonancia con lo hallado en otros trabajos publicados tanto en la población general española como entre los adolescentes durante toda la primera década del siglo XXI ${ }^{4,8-10,16}$. Hay importantes diferencias en la calidad de la dieta que reciben los 
escolares estudiados, estando relacionado con la clase social de la familia del menor y con el tipo de colegio al que acuden.

Por otra parte, los datos obtenidos del análisis de clúster y ofrecidos en el dendograma indican la existencia de una agregación de alimentos considerados saludables en unas familias y no saludables en otras, escogidos de forma agrupada a la hora de establecer la dieta familiar. De esta forma se comprueba que los alimentos considerados como menos saludables se agrupan en patrones de consumo y son más frecuentemente consumidos por clases sociales más bajas y entre aquellos escolares que acuden a centros escolares públicos.

Son numerosos los estudios ${ }^{17-19}$ que relacionan el bajo nivel de instrucción materna y el bajo nivel socioeconómico familiar con un mayor consumo de alimentos "no recomendables". Una menor accesibilidad a los alimentos más frescos o un menor conocimiento sobre las recomendaciones dietéticas en estas edades son dos de las causas que se podrían postular. Sin embargo, definir con precisión las motivaciones que contribuyen a la ingesta de los diferentes alimentos es difícil y está asociado a factores socioculturales y psicológicos como la edad, el género, la imagen corporal, el nivel socioeconómico familiar y las relaciones entre familiares e iguales $^{18,19}$.

Al comparar nuestros resultados con estudios previos realizados en la misma población ${ }^{8,14}$ encontramos un incremento en el consumo de derivados lácteos, zumos, refrescos, carnes magras embutidos y pescado, y una disminución de pan, arroz, patatas, hortalizas, legumbres y verduras, tal y como ocurre en general en la población española ${ }^{20}$. Así, es especialmente preocupante que menos de la mitad de los encuestados llegan a consumir las cantidades diarias recomendadas de productos lácteos, siendo estos resultados similares a los encontrados en otros estudios ${ }^{4,14,16}$.

En el grupo de carnes y pescados se encuentran hallazgos contradictorios. Un dato que acerca la realidad nutricional a la dieta mediterránea es la existencia de un consumo de carne de ave superior al de carne roja, al tiempo que la ingesta de pescado se ha igualado al de carne, algo que se refleja también en el Panel de Consumo Alimentario ${ }^{20,21}$. Dentro de este grupo de alimentos, el consumo de carne de ave aporta proteínas de alto valor biológico y el pescado, otro de los pilares de la alimentación mediterránea, es el principal suministrador de ácidos grasos poliinsaturados ${ }^{22}$. Sin embargo, los datos ofrecidos muestran una disminución en el consumo de estos alimentos, que pasan a tener una cadencia semanal mientras que no forman parte de la dieta habitual en un $20 \%$ del alumnado. Pese a que los embutidos no se consideran alimentos demasiado saludables por el gran contenido en grasas saturadas que aporta, casi dos tercios de los encuestados los consumen a diario.

El grupo de legumbres, tubérculos y frutos secos ha dejado de ser la base de la alimentación española ya que su consumo resulta más esporádico, confirmando su tendencia descendente ${ }^{23,24}$. Los frutos secos son poco empleados; aunque en principio serían deseables por su alto valor alimentario, éstos se ven limitados por el alto contenido en sal y grasa saturadas que presentan los productos comercializados. Respecto al pan, apreciamos un descenso progresivo de su ingesta en la sociedad española desde final del siglo pasado ${ }^{24}$ con un incremento del consumo de pan blanco y pan de molde.

El grupo de frutas y verduras es muy importante para el desarrollo físico del menor dado su aporte en fibras, tocoferoles, folatos, vitamina $\mathrm{C}$, beta-carotenos, fitoestrógenos y polifenoles ${ }^{6}$. Ninguna persona debería ingerir menos de 400 gramos de frutas frescas y verduras al día ${ }^{6}$ : esto se traduce en unas 2-4 piezas de frutas y de 3-5 raciones de verdura diarias. Sin embargo, casi un $10 \%$ de los encuestados no ingieren nada de fruta y menos de la mitad de los encuestados afirman tomar más de una pieza de fruta diaria; este porcentaje es similar al de la media europea para esa edad y a los países del área mediterránea ${ }^{1,4,20,21,25}$. Las verduras tienen un consumo incluso inferior al de la fruta, pues un tercio de los encuestados nunca las prueba. 
Dentro del grupo de las grasas y aceites, los adolescentes encuestados consumen mayoritariamente aceite de oliva, de forma muy superior a otros tipos de aceites como el de girasol. Por otra parte, el consumo de dulces y golosinas se ha incrementado respecto a encuestas previas ${ }^{8,14}$. Los datos del estudio muestran como casi cuatro de cada diez encuestados consumen golosinas a diario, con un elevado porcentaje de consumo de patatas embolsadas y comida rápida ${ }^{11}$, hábitos cuyo aprendizaje en la infancia favorece la continuación del consumo en la edad adulta (más de un $50 \%$ de la población andaluza los consume ${ }^{20}$. También se ha incrementado en la última década el consumo de bebidas con gas, que aumenta con la edad ${ }^{7,11}$, y el consumo de zumos no naturales, que tienen un alto contenido en azúcares. Diferentes estudios han demostrado una asociación entre el consumo de alimentos considerados como "calorías vacías", un mayor tiempo de ocio pasivo (televisión) y una reducción de la actividad física; todos ellos factores que favorecen un estilo de vida obesogénico y que son esenciales a tener en cuenta para la prevención de la adquisición de hábitos no saludables en edades tempranas ${ }^{26-28}$. Además, es en estas edades donde se pone de manifiesto una tendencia a incorporar patrones occidentales de hábitos alimentarios, con una mayor frecuentación de establecimientos de comida rápida en detrimento de una comida más elaborada.

El tipo de diseño mediante encuesta puede introducir un sesgo de memoria. El cuestionario utilizado, previamente validado, disminuye el error en la medición al preguntar sobre el consumo semanal y facilitar ejemplos fácilmente identificables por los menores ${ }^{14}$. Aunque el tipo de ingesta dietética experimenta variaciones temporales (día de la semana, estación del año...) se sustenta sobre un patrón de consumo básico que es el se intenta conocer mediante el cuestionario empleado. El muestreo estratificado según el carácter del colegio y los municipios junto al elevado número de participación e individuos permite una mayor garantía en la inferencia poblacional al resto de adolescentes escolarizados de nuestro entorno, aunque los resultados no serían totalmente extrapolables a otras sociedades con hábitos nutricionales diferentes o en aquellos adolescentes no escolarizados.

En líneas generales los hábitos alimentarios de los adolescentes estudiados se aproximan a un patrón de dieta saludable, sin embargo no cumplen las recomendaciones cualitativas sobre el consumo de los alimentos recomendables por grupos ${ }^{20}$. La adolescencia es un momento clave para la instauración de hábitos alimentarios saludables ya que en este periodo se asientan las bases para la alimentación adulta ${ }^{16}$. Las conductas alimentarias nacen en la familia, pueden reforzarse en el medio escolar y se contrastan en la comunidad en el contacto con pares y con el medio social ${ }^{5}$. Las características sociodemográficas como el nivel de estudios paternos y/o maternos podrían definir el estilo de vida de los encuestados y podrían condicionar el grado de adherencia al patrón de dieta recomendado ${ }^{17,29,30}$.

Teniendo en cuenta la relevancia de las intervenciones en la población más joven, se debe actuar de forma global sobre el entorno familiar y escolar, fomentando desde la primera etapa de la vida unos hábitos de alimentación y de vida saludables e inculcándoles su mantenimiento en la vida adulta $^{5}$. La puesta en marcha de estrategias de modificación de hábitos alimentarios en este rango de edad resulta esencial para conseguir mejorar la salud de la población adolescente y la futura población adulta.

\section{BIBLIOGRAFÍA}

1. Organización Mundial de la Salud. Obesidad y Sobrepeso. Nota descriptiva $n^{\circ} 311$. Ginebra. 2012 [consultado el 12/4/13]. Disponible en: http://www.who.int/mediacentre/factsheets/fs311/es/

2. Ministerio de Sanidad, Servicio Sociales e Igualdad. Encuesta Nacional de Salud 20112012. [Consultado el 15/04/2013] Disponible en: http://www.msc.es/estadEstudios/ estadisticas/encuestaNacional/encuestaNac2011/NotaTecnica2011-12.pdf

3. World Health Organization. Diet, Nutrition and the prevention of chronic diseases. Food and Agriculture Organization of the United Nations. Ginebra; 2003. 
4. Rubio Herrera MA, Gutiérrez Fuentes JA, Gómez de la Cámara A, Gómez Gerique JA. Evolución de la dieta española. Med Clin Monogr (Barc) 2011; 12: 35-36.

5. Ministerio de Sanidad y Consumo, Ministerio de Educación y Cultura, Ministerio del Interior. Nutrición saludable y prevención de los trastornos alimentarios 1999. (Consultado el 10 de abril de 2013). Disponible en: http://www.fhspereclaver.org/userfiles/file/ guia_nutricion_saludable.pdf

6. Pinto Fontanillo JA, Carbajal Azcona A. La dieta equilibrada, prudente o saludable. Dirección General de Salud Pública y Alimentación. Consejería de Sanidad y Consumo; 2006.

7. Moreno C, Ramos P, Rivera F, Jiménez-Iglesias A, GARCía I. Las conductas relacionadas con la salud y el desarrollo de los adolescentes españoles. Resumen del estudio Health Behaviour in School-aged Children (HBSC-2010). Ministerio de Sanidad, Servicios Sociales e Igualdad; 2012.

8. Instituto de Salud Carlos III. Alimentación y Valoración del Estado Nutricional en Adolescentes. Estudio Avena. Nutr Hosp 2003; XVIII: 15-28.

9. Serra Majem L, Ribas Barba L, Pérez Rodrigo C, Román Viñas, Aranceta Batrina J. Hábitos alimentarios y consumo de alimentos en la población infantil y juvenil española (19982000): variables socioeconómicas y geográficas. Med Clin (Barc) 2003; 121: 126-131.

10. GonzÁlez J. Comparación entre las preferencias alimentarias y el consumo alimentario de estudiantes universitarios. Nutr Hosp 2002; 22: 15-19.

11. Currie C, Gabhainn SN, Godeau E, Roberts C, Smith R, Currie D et al. Inequalities in young people's health: international report from the HBSC 2006 survey (Health Policy for Children and Adolescents). WHO Regional Office for Europe. Copenhagen; 2008.

12. REGIDOR E. La clasificación de la clase social de Goldthorpe: marco de referencia para la propuesta de la clase social del grupo de trabajo de la Sociedad Española de Epidemiología. Rev Esp Salud Publica 2001; 75: $13-22$.

13. Vázquez Martínez C, Alcaráz Cebrián F, Garriga García M, Martin E, Montagna MC, Ruperto MM et al. Dietética y nutrición: grupos de alimentos. Grupo Fisterra: Atención Primaria en la red. 2007. (Consultado el 18 de abril de 2013). Disponible en: http://www.fisterra. com/material/Dietetica/GruposAlimentos. asp
14. Mengual LuQue P. Estudio de los hábitos y conductas relacionados con la salud de los escolares de la ciudad de Córdoba, 1994/1999. [Tesis Doctoral]. Córdoba: Universidad de Córdoba. Facultad de Medicina; 2001.

15. Fundación Española de la Nutrición. Valoración de la dieta española de acuerdo al Panel de Consumo Alimentario. Ministerio de Medio Ambiente y Medio Rural y Marino; 2008. (Consultado el 5 de abril de 2013). Disponible en: http://www.mapa.es/alimentacion/ pags/consumo/dieta/valoracion_panel.pdf

16. Ayechu A, DurÁ T. Calidad de los hábitos alimentarios (adherencia a la dieta mediterránea) en los alumnos de educación secundaria obligatoria. An Sist Sanit Navar 2010; 33: 35-42.

17. Norte Navarro AI, Ortiz Monacada R. Calidad de la dieta española según el índice de alimentación saludable. Nutr Hosp 2011; 26: 330-336.

18. Aranceta Bartrina J, Pérez Rodrigo C, Ribas BarBa L, Serra Majem L. Epidemiología y factores determinantes de la obesidad infantil y juvenil en España. Rev Pediatr Aten Primaria 2005; 7: 13-20.

19. Cano Garcinuño A, Alberola lópez S, Casares Alonso I, Pérez García I. Desigualdades sociales en la prevalencia del sobrepeso y obesidad en adolescentes. An Pediatr (Barc) 2010; 73: 241-248.

20. Ministerio de agricultura, alimentación y medio ambiente. Valoración nutricional de la dieta española de acuerdo al panel de consumo alimentario. 2012. (Consultado el 5 de abril de 2013). Disponible en: http://www.fen. org.es/imgPublicaciones/30092012125258. pdf

21. Díez Gañan L, Galán labaca I, León Domínguez CM, ZorRILla ToRRAS B. Ingesta de alimentos, energía y nutrientes en la población de 5 a 12 años de la comunidad de Madrid: Resultados de la encuesta de nutrición infantil 2001-2002. Rev Esp Salud Publica 2007; 81: 543-545.

22. González Gross M, Gómez Lorente JJ, Valtueña J, Ortíz JC, MELÉndez A. "La pirámide del estilo de vida saludable" para niños y adolescentes. Nutr Hosp 2008; 23: 159-168.

23. GASCÓn JimÉnEZ FJ. Estudio de los hábitos y conductas relacionados con la salud de los escolares de la provincia de Córdoba, excluida la capital [Tesis Doctoral]. Córdoba: Universidad de Córdoba. Facultad de Medicina; 1995. (Consultado el 10 de diciembre de 2012). Disponible en: http://whqlibdoc.who. int/trs/who_trs_916.pdf 
24. Pérula de Torres L. Estudio de los hábitos y conductas relacionados con la salud autopercibida [Tesis Doctoral]. Córdoba: Universidad de Córdoba. Facultad de Medicina; 1995.

25. Moreno C, Muñoz-Tinoco V, Pérez P, SánchezQueija I, Granado MC, Ramos P et al. Desarrollo adolescente y salud. Resultados del estudio HBSC 2006 con chicos y chicas españoles de 11 a 17 años. Ministerio de Sanidad y Consumo. Madrid; 2008.

26. Salmon J, Campbell KJ, CRaWford DA. Television viewing habits associated with obesity risk factors: a survey of Melbourne schoolchildren. Med J Aust 2006; 184: 64-67.

27. Dubois L, Farmer A, Girard M, Peterson K. Social factors and television use during meals and snacks is associated with higher BMI among pre-school children. Public Health Nutrition 2008; 11: 1267-1279.

28. Amigo Vazquez I, Busto Zapico R, Fernández RoDRíGUEz C. La obesidad infantil como resultado de un estilo de vida obesogénico. Endocrinol Nutr 2007; 54: 530-534.

29. González CA, Argilaga S, Agudo A, Amiano P, Barricarte A, Beguiristain JM et al. Diferencias sociodemográficas en la adhesión al patrón de dieta mediterránea en poblaciones de España. Gac Sanit 2002; 16: 214-221.

30. González Jiménez E, Aguilar Cordero MJ, García García CJ, García López P, Álvarez Ferre J, Padilla López A et al. Influencia del entorno familiar en el desarrollo del sobrepeso y la obesidad en una población de escolares de Granada (España). Nutr Hosp 2012; 27: 177184. 